\title{
A Decision Support System to Evaluate the Competitiveness of Nations
}

\author{
Ş.ÖNSEL ${ }^{\mathrm{a}}$, F.ÜLENGINN ${ }^{\mathrm{a}}$, G.ULUSOY ${ }^{\mathrm{b}}$, Ö.KABAK ${ }^{\mathrm{c}}$, Y.İ.TOPCU $^{\mathrm{c}}$, E.AKTAŞ ${ }^{\mathrm{c}}$ \\ ${ }^{a}$ Dogus University, Faculty of Engineering, Department of Industrial Engineering, Acibadem, 34722, \\ Kadikoy, Istanbul, TURKEY \\ ${ }^{\mathrm{b}}$ Sabancı University, Faculty of Eng. and Natural Sciences, Orhanli, Tuzla, 81474 Istanbul, TURKEY \\ ${ }^{\mathrm{c}}$ Istanbul Technical University, Faculty of Management, Department of Industrial Engineering, \\ Macka, 34357, Istanbul, TURKEY
}

\begin{abstract}
The measurement of competitiveness and strategy development is an important issue for policy makers. The aim of this paper is to explore methodological transparency as a viable solution to problems created by existing aggregated indices as well as to conduct a detailed analysis on the ongoing performance of nations' competitiveness. For this purpose, a methodology composed of three steps is used. To start, a combined clustering analysis methodology is used to assign countries to appropriate clusters. In current methods, country clustering is generally based on GDP. However, we suggest that GDP alone is insufficent to define the stage of competitiveness a country belongs. In the proposed methodology, 135 criteria are used for a proper classification of the countries. Relationships between the criteria and classification of the countries are determined using Artificial Neural Networks (ANNs). ANN provides an objective method for determining the attribute/criteria weights, which are, for the most part, subjectively specified in existing methods. Finally, in the third step, the countries of interest are ranked based on weights generated in the previous step. Beyond the ranking of countries, the proposed methodology can also be used to identify those attributes that a given country should focus on in order to improve its position relative to other countries, i.e., to transition from its current cluster to the next higher one. As a final analysis, the dynamic change of the rank of the countries over years has also been investigated.
\end{abstract}

Key-Words: - Ranking, Competitiveness, Artificial Neural Network, Cluster Analysis

\section{Introduction}

A nation's competitiveness can be viewed as its position in the international marketplace compared to other nations of similar economic development. The capability of firms to survive and to have a competitive advantage in global markets depends, among other things, on the efficiency of their nation's public institutions, excellence of the educational, health and communication infrastructures, as well as the nation's political and economical stability. On the other hand, an outstanding macroeconomic environment alone cannot guarantee a high level of national competitive position unless firms create valuable goods and services with a commensurately high level of productivity at the micro level. Therefore, the micro and macroeconomic characteristics of an economy jointly determine its level of productivity and competitiveness.

Although many view competitiveness as a synonym for productivity [1], these two related terms are, in fact, different. Productivity refers to the internal capability of an organization while competitiveness refers to the relative position of an organization vis-à-vis its competitors. Each year, some organizations, such as the World Economic Forum (WEF) [3], and the Institute for Management Development (IMD) [2], publish rankings of national competitiveness among countries. These rankings serve as benchmarks for national policy makers and interested parties in judging the relative success of their countries in achieving competitiveness as represented by well-known and accepted indices. However, for the last quarter-century, the WEF has led in evaluation of the competitiveness of nations through its publication, The Global Competitiveness Report [3].

With 2006-2007 Report [3], WEF decided to use the Global Competitiveness Index (GCI) [6], as the main competitiveness indicator. The GCI, albeit simple in structure, provides a holistic overview of factors that are critical to driving productivity and competitiveness, and groups them into nine pillars that are different from the 2004-2005 report [4] where 12 pillars are assumed. Combining some of the pillars and separating a pillar results with such a decrease.

The nine pillars are measured using both hard data from public sources (such as inflation, internet penetration and school enrolment rates) and data from the World Economic Forum's Executive Opinion Survey, which is conducted annually among top executives in all of the countries assessed. The survey provides crucial data on a number of qualitative issues (e.g. corruption, confidence in the public sector, quality of schools ) for which no hard data exist.

In addition to the change in the number of pillars, 
there are also changes on the variable configuration and structure of the pillars. In the recent report total number of variables are decreased from 177 to 137.

Although there are changes in the pillars and variables in the 2006-2007 report, the basic approach to the evaluation procedure remains unchanged. The pillars are still used as sub-index for three main dimensions of competitiveness: Basic requirements (the first 4 pillars), efficiency enhancers $\left(5^{\text {th }}\right.$ to $7^{\text {th }}$ pillars), and innovation and sophistication factors (last 2 pillars).

An important characteristic of the GCI is that it explicitly takes into account the fact that the countries around the world are at different levels of economic development. What is important for improving the competitiveness of a country at a particular stage of development will not necessarily be the same for a country in another stage. Thus GCI separates countries into three specific stages: factor-driven, efficiency driven and innovation-driven. Therefore, in the calculation of the final GDI; the weights of the three dimensions are determined according to the stage that country belongs[3]. Unfortunately, this classification tends to be rather subjective, or is based solely on per capita income. Subjectivity is also present when creating the threshold used to separate one stage from another. Some degree of objectivity is possible, however, if countries are clustered as a function of their similarities on selected criteria. By doing so, important factors underlying the competitiveness position of each stage, and of particular countries at various stages, can be revealed. It will thus be easier to understand the internal dynamics of each stage, and to provide useful and objective guidelines to countries as they attempt to improve their positions with respect to those located at higher stages.

Section 2 of this paper introduces our proposed methodology to cluster countries into stages, and to generate criteria weights that are critical at each stage of the procedure. In Section 3, a composite index is calculated using the calculated weights. The results are then compared to those of the Global Competitiveness Index of the WEF to determine whether the weights adopted by the WEF incorrectly penalize some countries and/or reward others. This section also provides some useful guidelines to selected countries as they seek to improve their relative competitiveness. Besides, in this section the change of the nations' competitiveness rank is analysed also. The paper closes with conclusions and suggestions for further improvements of the proposed methodology.

\section{Proposed Methodology}

The aim of this research is, first, to provide an objective clustering of countries according to their values/scores on selected criteria, second, to propose an objective weighting procedure to calculate an aggregated index. For these purposes, a three-step methodology is proposed. Finally the results are compared with our previous study's [5] findings to track the changes in the competitiveness of the countries between 2005 and 2007.

The proposed methodology considers 135 criteria in the clustering process. The criteria are the hard data and survey data used in the WEF report [3]. Two of the criteria have been removed from considiration due to the lack of available data.

In particular, a hierarchical cluster analysis is used to determine the "best" number of clusters; this number is then used as a parameter to determine the appropriate clusters of countries using Self-Organizing Maps [8]. Next, relationships between the criteria and the classification of countries are determined using in an objective manner using Artificial Neural Networks (ANN). Importantly, existing methodologies generally assess criteria weights/importances subjectively. Finally, in the third step of our procedure, countries are rankordered based on the ANN-generated weights and the dynamic change in the rank of countries is analysed. The proposed methodology can also be used to identify those attributes a country should focus on in seeking to improve its position relative to other countries. i.e., to transition from its current cluster to the better one.

\subsection{Classification of Countries}

In the first part of this research, countries are grouped based on their similarity of characteristics. Cluster analysis, is used for this purpose.

\subsubsection{Cluster Analysis}

Cluster analysis involves grouping similar objects into mutually exclusive subsets referred to as clusters [8]. The cluster definition problem is NP-complete, so a computationally efficient, exact solution method, to the best of the authors' knowledge, does not exist. However, a number of heuristic methods have been proposed for this purpose, including agglomerative techniques [8]. All hierarchical agglomerative heuristics begin with $\mathbf{n}$ clusters, where $\mathbf{n}$ is the number of observations. Then, the two most similar clusters are combined to form $\mathbf{n - 1}$ clusters. On the next iteration, $\mathbf{n}-\mathbf{2}$ clusters are formed with the same logic, and this process continues until one cluster remains. Only the rules used to merge clusters differ across the various heuristics.

In order to improve the accuracy of, and reduce any subjectivity in, the cluster analsyis, we employ a SelfOrganizing Map (SOM) Neural Network, as suggested by Mangiameli et al [9]. The SOM is thus not taken as an alternative, but rather as a complementary analysis 
that follows hierarchical clustering. The focus is on improved accuracy in the assignment of observations to appropriate clusters, given that the number of clusters in the data is known. The SOM's network learns to detect groups of similar input vectors in such a way that neurons physically close in the neuron layer respond to a similar input vector [10].

\subsubsection{Determining the Country Clusters}

The basic drawback of any study based solely on ranking is that the ordinal scale does not reflect the appropriate competitiveness level of a country relative to other countries. The most accurate position of a country within the total configuration can only be determined after the grouping of nations is performed, and similarities to the evaluated country in terms of competitiveness are identified.

In the current study, the Ward hierarchical method, an agglomerative clustering technique, and the Euclidean distance measure, were selected as most appropriate based on evaluations using MATLAB [11]. In Ward's method, the distance is the ANOVA sum of squares between two clusters summed over all variables [8]. An analysis of the dendogram and ANOVA were thus used to test the significance of differences between the cluster means, producing three significant clusters. Dendrogram analysis generates a dendrogram plot of the hierarchical, binary cluster tree. It consists of many U-shaped lines connecting objects in a hierarchical tree. The height of each $U$ represents the distance between the two objects being connected. Each leaf in the dendrogram corresponds to one data point. As can be seen from Figure 1, the countries can be grouped into three different U-shaped clusters according to 2006-2007 data.

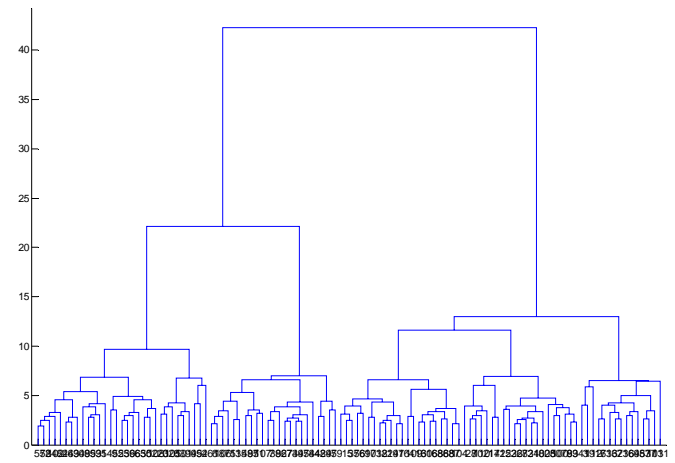

Figure 1 Dendogram of the country clusters

Next, the appropriate number of clusters generated in the first stage was used to repeat the analysis using SOM and MATLAB software. Since we sought to categorize the countries into three classes, there were three outputs in the ANN's configuration. This generated a $3 * 1$ matrix of the weight vector. The topology function used was "HEXTOP," which means that the neurons were arranged in a hexagonal topology at the Kohonen layer, while the distance function was "MANDIST," i.e., the Manhattan (city block) distance. The training of a selforganizing map using MATLAB involved two steps: Ordering phase and tuning phase. In the former, the ordering phase learning rate and neighborhood distance are decreased from that rate and maximum distance between two neurons to the tuning phase learning rate and tuning phase neighborhood distance, respectively. The ordering phase lasts for a given number of steps. At the tuning phase, the learning rate is decreased much more slowly than is the ordering phase, while the neighborhood distance stays constant [12]. In the current study, the ordering phase learning rate, ordering phase steps, and tuning phase learning rate were taken as 0.9 , 1000 , and 0.02 , respectively. The countries contained within the resulting clusters are determined by the end of this first stage of the method. The resulting clusters as well as the related countries that are found by 2006 data are given in the Table 1.

Table 1 Clusters of Countries

\begin{tabular}{|l|l|}
\hline \multicolumn{2}{|l|}{ HIGHLY COMPETITIVE (HC) COUNTIRES: } \\
\hline HC in 2004: Australia; & CO in 2004: Chile; Czech \\
\hline Austria; Belgium; Canada; & Republic; Estonia; India; Korea, \\
Denmark; Finland; France; & Rep.; Malaysia; Portugal; \\
Germany; Iceland; Ireland; & Slovenia; Spain; Tunisia; United \\
Israel; Japan; Luxembourg; & Arab Emirates \\
Netherlands; New Zealand; & \\
Norway; Singapore; & \\
Sweden; Switzerland; & \\
Taiwan; UK; US & \\
\hline COMPETITIVE (CO) CoUNTRIES: \\
\hline CO in 2004: Bahrain; & NC in 2004: Colombia; Croatia; \\
\hline Brazil; Costa Rica; Cyprus; & El Salvador; Jamaica; Mexico; \\
Egypt; Greece; Hungary; & Panama; Poland; Turkey; \\
Indonesia; Italy; Jordan; & Uruguay \\
Kuwait; Lithuania; Malta; & \\
Mauritius; Slovak Republic; & \\
South Africa; Thailand & \\
\hline NON-COMPETITIVE (NC) COUNTRIES: \\
\hline CO in 2004: Botswana; & NC in 2004: Algeria; Angola; \\
\hline China; Morocco; Namibia & Argentina; Bangladesh; Bolivia; \\
& Bosnia and Herzegovina; \\
& Bulgaria; Chad; Dominican \\
& Republic; Ecuador; Ethiopia; \\
& Gambia; Georgia; Guatemala; \\
& Honduras; Kenya; Macedonia; \\
& Madagascar; Malawi; Mali; \\
& Mozambique; Nicaragua; \\
& Nigeria; Pakistan; Paraguay; \\
& Russian Federation; Serbia and \\
& Montenegro; Sri Lanka; \\
& Uanzania; Trinidad and Tobago; \\
& Vietnam; Zambia; Zimbabwe \\
\hline
\end{tabular}


Comparison of the results with our previous findings [5] shows that Turkey, our home country, has shown an impressive improvement in the competitive performance moving up from the non-competitive to competitive countries. Colombia, Croatia, El Salvador, Jamaica, Mexico, Panama and Poland and Uruguay have also shown a similar transition. However, China, Morocco, Namibia, Botswana have moved from the competitive to non-competitive stage. Finally; Chile, Check Republic, Estonia, India, Korea, Malaysia, Portugal, Slovenia, Spain, Tunisia, United Arab Emirates moved up from the competitive to highly competitive stages. None of the countries previously assigned to highly competitive cluster moved down to a lower stage.

\subsection{Identification of Basic Criteria Underlying Country Stages through ANN}

At this step of the study, the basic factors underlying the reasons a country belongs to a specific cluster is analyzed using ANN. The feed-forward back propagation algorithm is used for this purpose.

\subsubsection{Artificial Neural Networks}

ANN techniques have been applied to a variety of problem types and, in many instances, provided superior results to conventional methods [13]. The literature [e.g., $14,15,16]$ suggests the potential advantages of ANN versus classical statistical methods. The basic ANN model consists of computational units that emulate the functions of a nucleus in a human brain. The unit receives a weighted sum of all its inputs and computes its own output value by a transformation, or output, function. The output value is then propagated to many other units via connections between units. The learning process of ANN can be thought of as a reward and punishment mechanism [17]. When the system reacts appropriately to an input, the related weights are strengthened. As a result, it becomes possible to generate outputs, which are similar to those of the previously encountered inputs. In contrast, when undesirable outputs are produced, the related weights are reduced. The model will thus learn to give a different reaction when similar inputs occur. In this way, the system is "trained" to produce desirable results while "punishing" undesirable ones.

In multilayer networks, all inputs are related to outputs through hidden neurons - i.e., there is no direct relationship among them. As a result, specification of the characteristics of each input neuron and the strength of relation between input $X_{i}$ and output $O_{i}$ can be found using the method proposed by Onsel et al. [7]:

$$
R S_{j i}=\frac{\left[\sum_{k=0}^{n}\left(W_{k i} * U_{j k}\right)\right]^{2}}{\sum_{i=0}^{m}\left[\sum_{k=0}^{n}\left(W_{k i} * U_{j k}\right)\right]^{2}}
$$

In this expression, $R S_{j i}$ represents the strength of relation between input $i$ and output $\mathrm{j}$. $W_{k i}$ is the weight between the $j^{\text {th }}$ output $U_{j k}$ and the $k^{\text {th }}$ hidden neuron. $R S_{j i}$ is thus the ratio of the strength of relation between the $i^{\text {th }}$ input and $j^{\text {th }}$ output to the sum of all such strengths. The absolute value in the denominator is used to avoid positive relations cancelling the impact of negative ones.

\subsubsection{Determining Basic Criteria Weights}

Output from the SOM in the previous stage helps generate the clusters of countries. These data are then used as the output of the multilayer feed-forward ANN while the 135 criteria are treated as inputs.

68 countries are used for training, 22 countries for validation and again 22 countries for testing stages. In order to obtain robust results based on different trials, for each hidden neuron number, the ANN is computed ten times, and the best results obtained from each taken. In this way, an attempt is made to detect different points of weight space corresponding to the network via several experiments. The optimal hidden neuron number is found as 5 . The tangent sigmoid function (tansig) is used to show the relation between the input-hidden and the hidden-output layers. The training algorithm is a gradient-descent method with momentum and an adaptive learning ratio ("traingdx"). The validation vectors are used to stop training early if further training on the primary vectors will hurt generalization to the validation vectors [12]. Test vector performance can be used to measure how well the network generalizes beyond primary and validation vectors. The mean square error, selected as the performance measurement, was found to be 0.00017 . The importance of the imputs (criteria), playing the dominant role in allocation of countries to the three clusters, were obtained using the modified Onsel et al. [7] formula. The the most important five criteria in each cluster is as follows:

High Competitive Countries: Inflation, Local equity market access, Reliance on professional management, Personal computers, Local supplier quantity

Competitive Countries: Judicial independence, Pervasiveness of illegal donations to political parties, Medium-term business impact of tuberculosis, Mediumterm business impact of malaria, Informal sector

Non-Competitive Countries: Local equity market access, Favoritism in decisions of government officials, Degree of customer orientation, Local supplier quantity, Pay and productivity 


\section{Ranking Countries Based on the Proposed Weighted Criteria Index}

At the third step of this research, the weights of 135 criteria for each cluster calculated in the previous step are used to rank the countries. For this purpose, initially, the weights are normalized. The score obtained by each country from each of the criteria is then multiplied by the normalized weight of that criterion. The 112 countries are subsequently ranked according to these weighted index values.

The top 10 ranked countries are (in the order of rank) Switzerland, Finland, Denmark, Germany, Sweden, Singapore, Japan, Netherlands, Hong Kong SAR, UK, Austria, and US.

The rankings found with the proposed approach are compared with that obtained with 2004-2005 data. This dynamic comparison will also show which countries have dealt with the key determinants of competitiveness at their level of development such as macroeconomic stability or education and health etc. It is also be possible to underline the additional factors over which the countries should focus in order to switch to higher clusters of develeopment.

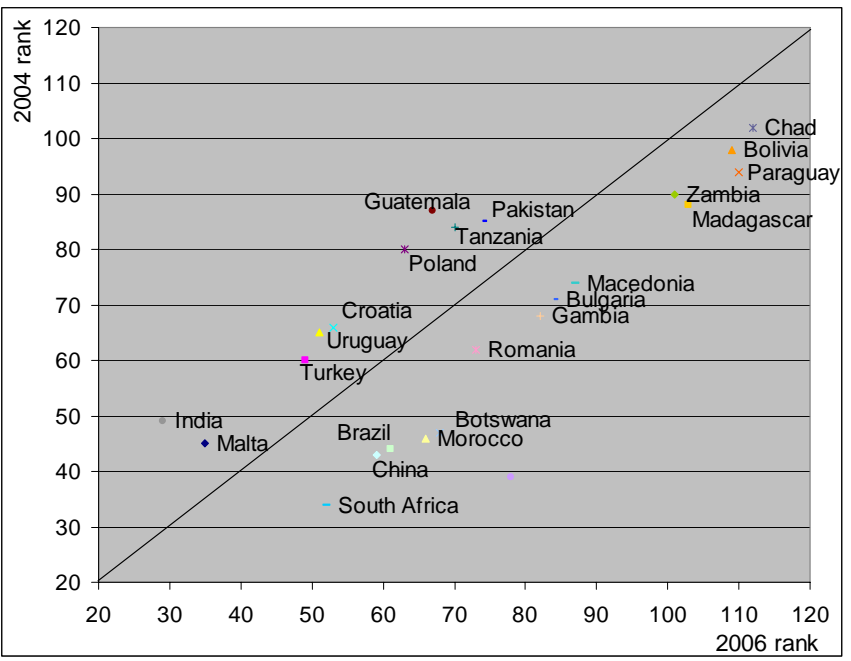

Figure 2. Countries that show important changes in ranking (2004-2006)

In Fig. 2, the countries that have an improvement or decline by ten points with respect to 2005 ranking can be seen. Accordingly, India had the most dramatic improvement moving from $49^{\text {th }}$ to $29^{\text {th }}$ in ranking. Poland $(+17)$ and Guatemala $(+20)$ constitute the other countries having the most important improvement in ranking. Besides, our home country, Turkey, moving from $49^{\text {th }}$ to $60^{\text {th }}$ is among the most improved seven countries.
On the other hand Nabia is a country which shows a dramatic decrease with respect to previous analysis ranking (from $78^{\text {th }}$ to $39^{\text {th }}$ ). It is interesting to note that China (-17) and Brazil (-16) which are accepted as emerging countries as well as Bulgaria which has recently accessed EU countries are among countries with worst competitiveness performance change with respect to 2004 ranking.

\section{Conclusion and Further Suggestions}

Despite attempts to provide objectivity in the development of indicators for the analysis of the competitiveness of countries, there are obviously subjective judgments about how data sets are aggregated and what weighting is applied. Generally, either equal weighting is applied to calculate the final index or subjective weights are specified. The same problem also occurs in the subjective assignment of countries into different clusters. For example the WEF assigns countries to different stages of development mainly on the basis of their GDP level and the application of different subjective weights for each stage. These subjectivities may create a bias, as selecting specific data simultaneously overestimates the level of competitiveness of some countries, making them look unrealistically good, while underestimating that of others.

It is important to emphasize that the subjectivity of the WEF clustering, as well as of the weighting process, sometimes result in contradictory results with respect to the WEF's index. In particular, important discrepancies may occur between the stage to which a country is assigned and the rank that it receives based on the GCI. When a country is assigned to a stage, logically, it is not expected to be ranked lower than the countries in worst stages nor higher than the ones in better stages. Therefore, developed as such, these types of indices do not provide useful guides for the executives and policy makers.

The aim of this paper is to explore whether methodological transparency can be an adequate solution to the above-given problems posed by the current aggregated indices. For this purpose, a methodology is proposed to objectively group countries into clusters as well as to specify the weight of the criteria that play the dominant role in each cluster. A new composite index that uses calculated weights has been created. By doing so, the criticism that it is simply an attempt to make some countries more competitive than they actually are can be avoided. What's more, by focusing on the criteria necessary to move a country into a higher cluster, the index can be used by both policymakers and executives responsible for making their countries more competitive. 
Moreover, the dynamic structure of the changes in the rankings of the countries' competitiveness level is also analysed in detail.

As a further study a panel data analysis can be conducted in order to see the evolution of competitiveness of the countries.

\section{References:}

[1] M. Oral, U. Cinar, H. Chabchoub. Linking industrial competitiveness and productivity at the firm level. European Journal of Operational Research 118(2), 1999 271-277.

[2] http://www.imd.ch

[3] WEF: The Global Competitiveness Report, 20062007, Hampshire: Palgrave Macmillan, 2006

[4] WEF: The Global Competitiveness Report, 20042005, Hampshire: Palgrave Macmillan, 2004

[5] Ş. Onsel, F.Ulengin, G.Ulusoy, E.Aktaş, Ö.Kabak, Y.İ.Topcu. A new perspective on competitiveness of nations, Socio-Economic And Planning Science (in press), 2007

[6] X. Sala-i-Martin, E.V. Artadi. The Global Competitiveness Index. In The Global Competitiveness Report 2004-2005. Hampshire: Palgrave Macmillan. 51-80, 2004

[7] S. Onsel Sahin, F. Ulengin, B. Ulengin., A dynamic approach to scenario analysis: the case of Turkey's inflation estimation. European Journal of Operational Research 158(1), 2004, 124-145

[8] J. Hair, K.E. Anderson, W.C. Black. Multivariate Data Analysis with Readings. Prentice Hall, New York, 1995

[9] P. Mangiameli, S.K. Chen, D.A. West. Comparison of SOM neural network and hierarchical clustering. European Journal of Operational Research 93(2), 1996, 402-417.

[10] T. Kohonen. Adaptive associative and selforganizing functions in neural computing. Applied Optics 26(23), 1987, 4910-4918

[11] http://www.mathworks.com

[12] http://www.mathworks.com/access/helpdesk/help/ pdfdoc/nnet/nnet.pdf

[13] Y. Yoon, G. Swales, T.M. Margavio. A comparison of discriminant analysis versus artificial neural networks.Journal of Operational Research Society 44(1), 1993, 51-60

[14] M. Boznar, M. Lesjak, P. Mlakar. A neural network-based method for short-term predictions of ambient $\mathrm{SO} 2$ concentrations in highly polluted industrial areas of complex terrain. Atmospheric Environment B: Urban Atmosphere 27B, 1993, 221230
[15] H.B. Hwarng, H.T. Ang. A simple neural network for ARMA (p,q) time series. Omega 29, 2001, 319333

[16] N. R. Swanson, H. White. Forecasting economic time series using flexible versus fixed specification and linear versus nonlinear econometric models. International Journal of Forecasting 13(4), 1997, 439-461

[17] H. Hruschka. Determining market response functions by neural network modeling: a comparison to econometric techniques, European Journal of Operational Research 66(1), 1993, 27-35 